\title{
Crecimiento de los pastos Cayman y Cobra en diferentes niveles salinos de $\mathrm{NaCl}$, en invernadero \\ Greenhouse growth of Cayman and Cobra grasses under diverse $\mathrm{NaCl}$ salinity levels
}

\author{
Edgar Iván Sánchez-Bernal ${ }^{1 *}{ }^{\circledR}$, Silvia Santos-Jerónimo2 ${ }^{(\mathbb{D}}$, Héctor Manuel Ortega-Escobar $^{3}$ (1), \\ Serafín Jacobo López-Garrido ${ }^{2}$ (1) y Marco Antonio Camacho-Escobar $^{4}$ (1)
}

\footnotetext{
${ }^{1}$ Instituto de Ecología, ${ }^{2}$ Posgrado en Producción y Sanidad Animal, ${ }^{4}$ Cuerpo Académico Ciencias Agropecuarias, Universidad del Mar, Campus Puerto Escondido. Carretera Vía Sola de Vega km 1.5 Puerto Escondido. 71980 San Pedro Mixtepec, Oaxaca, México.

* Autor para correspondencia (edgarivansb@zicatela.umar.mx)

${ }^{3}$ Profesor Investigador Titular Postgrado en Hidrociencias, Colegio de Postgraduados, Campus Montecillo. Carretera Federal México-Texcoco km 36.5, Montecillo. 56230 Texcoco, Estado de México, México.
}

\section{RESUMEN}

Los suelos salinos afectan el crecimiento y rendimiento de los pastizales y disminuyen la disponibilidad de forrajes para la alimentación animal. Objetivo: Evaluar la emergencia, crecimiento y producción de biomasa de los pastos Cayman $(\mathrm{BR} / 1752)$ y Cobra $(\mathrm{BR} / 1794)$ en condiciones de estrés salino inducido por la sal $\mathrm{NaCl}$ a siete niveles de conductividad eléctrica (CE): $0,2,4,6,10$, 12 y $15 \mathrm{dS} \mathrm{m}^{-1}$. Metodología: en invernadero, se establecieron macetas experimentales con agrolita, sembradas a una densidad de 25 semillas por maceta para evaluar la emergencia; posteriormente se realizó un aclareo para dejar tres plantas por maceta en un diseño completamente al azar. El riego consistió en reponer el agua perdida por evapotranspiración, mediante el suministro de agua destilada. El experimento abarcó un periodo de 60 días durante los cuales se determinó la altura y el diámetro del tallo, número de hijuelos, el número de hojas, la longitud de raíz y producción de biomasa. Los resultados indican que al aumentar la conductividad eléctrica de las soluciones salinas, ambos pastos disminuyeron de manera diferencial el porcentaje de emergencia, la altura y el diámetro del tallo, el número de hijuelos y el número de hojas, con una disminución en la producción de biomasa de las plantas, más pronunciado en pasto Cayman que en pasto Cobra. Entre cultivares se presentaron diferencias de tolerancia; ya que en el pasto Cayman

Cita recomendada:

Sánchez-Bernal, E. I., S. Santos-Jerónimo, H. M. Ortega-Escobar, S. J. López-Garrido y M. A. Camacho-Escobar. 2020. Crecimiento de los pastos Cayman y Cobra en diferentes niveles salinos de $\mathrm{NaCl}$, en invernadero. Terra Latinoamericana Número Especial 38-2: 391-401.

DOI: https://doi.org/10.28940/terra.v38i2.613 esta disminución se produjo a $\mathrm{CE} \geq 10 \mathrm{dS} \mathrm{\textrm {m } ^ { - 1 }}$, mientras que en el pasto Cobra la producción de biomasa fue afectada significativamente $(P=0.05)$ a partir de CE $\geq 12 \mathrm{dS} \mathrm{m}^{-1}$.

Palabras clave: crecimiento, $\mathrm{NaCl}$, pastos, producción de biomasa, tolerancia a sales.

\section{SUMMARY}

Saline soils affect the growth and yield of pastures and decrease the availability of forage for animal feed. Objective: To evaluate the emergence, growth and biomass production of the Cayman (BR/1752) and Cobra (BR/1794) grasses under salinity conditions induced by $\mathrm{NaCl}$ salt at seven levels of electrical conductivity (CE) of $0,2,4,6,10,12$ and $15 \mathrm{dS} \mathrm{m}^{-1}$. Methodology: In a greenhouse, experimental pots with agrolite were planted at a density of 25 seeds per pot, to evaluate emergence. Later a clear was performed to leave three plants per pot in a completely random design. Irrigation consisted of replacing the water lost through evaporation by supplying distilled water. The experiment covered a period of 60 days, during which the height of plants, diameter of stem, number of shoots, number of leaves, root length and biomass production were determined. The results indicate that increasing the concentration of $\mathrm{NaCl}$ for both pastures, differentially decreased the percentage of emergence, height of plants, stem diameter, the number of shoots 
and the number of leaves and consequently these reductions affected the biomass production of the plants. The degrowth was more pronounced in Cayman grass than in Cobra grass. Cultivars showed tolerance differences since in the Cayman grass biomass production was significantly affected $(P=0.05)$ at $\mathrm{CE} \geq 10 \mathrm{dS} \mathrm{m}^{-1}$, while in Cobra grass this decrease occurred at $\mathrm{CE} \geq 12 \mathrm{dS} \mathrm{m}^{-1}$.

Index words: growth, $\mathrm{NaCl}$, pasture, biomass production, salinity tolerance.

\section{INTRODUCCIÓN}

La salinidad de los suelos limita la productividad de las plantas cultivadas para la alimentación humana y animal (Mesa, 2003). En México, el 10\% del área irrigada está afectada por salinidad (Ruiz-Cerda et al., 2007). El estado de Oaxaca presenta este problema, y en la zona costera por sus características edafo-climáticas se incrementa. Estudios realizados (Sánchez-Bernal et al., 2012a, b; 2014) en el valle costero de Oaxaca indican suelos afectados en su capa superior principalmente por las sales cloruro de sodio $(\mathrm{NaCl})$, cloruro de magnesio $\left(\mathrm{MgCl}_{2}\right)$ y bicarbonato de sodio $\left(\mathrm{NaHCO}_{3}\right)$; de éstas, el $\mathrm{NaCl}$ es predominante. La elevada concentración de sales de estos suelos se debe a procesos naturales y antropogénicos; naturales como intrusiones marinas, deposición de la sal a tierras cercanas por acción del viento, un deficiente drenaje natural de los suelos, una capa freática somera; antropogénicos como la deforestación, cambio de uso del suelo, sobrepastoreo, riego con agua de elevada concentración salina y mal uso de fertilizantes químicos (Cisneros et al., 2003 '; Sánchez-Bernal et al., 2019). Los suelos salinos presentan $\mathrm{CE}>4 \mathrm{dS} \mathrm{m}^{-1}, \mathrm{pH}$ de 7.3 a 8.5 y menos de $15 \%$ de sodio intercambiable que limitan el crecimiento y desarrollo de los cultivos incluyendo especies forrajeras (Martínez et al., 2011). Al respecto Ruiz-Ramírez et al. (2012) reportan que la salinidad disminuyó la germinación y vigor de las semillas de los pastos Buffel (Cenchrus ciliaris L.), Rhodes (Chloris gayana L.), Toledo (Brachiaria brizantha (Hochst. Ex A. Rich.)) Stapf, Mulato II (Brachiaria hibrido) y Tanzania (Panicum máximum Jacq). Otros investigadores como Bizhani y Salehi (2014) observaron menor tamaño de los tallos, peso seco y fresco de las plantas, área foliar, tasa fotosintética y contenido de clorofila en pasto Bermuda común (Cynodon dactylon L.) y en pasto Azul de kentucky (Poa pratensis L.). Por otra parte, en Raigrás perenne (Lolium perenne L.) se registró un aumento en la mortalidad de plantas; y una disminución de la tasa de germinación, la longitud de la raíz y el tallo, el número de hojas, así como en el peso seco y fresco de las plantas (Nizam, 2011). En general, el estrés abiótico salino disminuye el crecimiento y producción de biomasa de los pastos, en consecuencia, aminora la disponibilidad de forraje para la alimentación animal y afecta directamente a la actividad económica ganadera. Una de las medidas que permiten aprovechar los suelos degradados por las altas concentraciones de sales es la utilización de pastos tolerantes, los cuales constituyen una alternativa para el desarrollo sostenible de pastizales costeros (Masters et al., 2007; Cruz et al., 2017). En este trabajo se evaluó la emergencia, crecimiento y producción de biomasa de los pastos híbridos del género Urochloa Cayman (BR/1752) y Cobra (BR/1794), sometidos a 6 niveles de salinidad inducida por $\mathrm{NaCl}$ más un testigo, para determinar sus niveles de tolerancia a esta sal, presente en la llanura costera de Oaxaca.

\section{MATERIALES Y MÉTODOS}

\section{Área de Estudio}

Se estableció un experimento de tolerancia a la sal $\mathrm{NaCl}$ de pastos Cayman y Cobra, en condiciones de invernadero, en el Centro de investigación de pastos tropicales, propiedad de la Empresa Semillas Papalotla, S. A. de C.V., en Santa Elena, Municipio de Santa María Tonameca, Oaxaca ( $15^{\circ} 44^{\prime} 20.53$ ” N, $96^{\circ} 50^{\prime} 45.77^{\prime}$ O). Se trata de una zona de llanura costera en la que la vegetación de selva baja caducifolia fue eliminada para al establecimiento de ganadería extensiva. El clima de la región es cálido subhúmedo $\mathrm{Aw}_{2}$, con una precipitación media anual de $1213 \mathrm{~mm}$ y una temperatura media anual entre los 24 y $27.2{ }^{\circ} \mathrm{C}$ (Serrano et al., 2005).

\section{Tratamientos y Diseño Experimental}

Se evaluaron 7 tratamientos conformados por 6 niveles de salinidad, $0,2,4,6,10,12$ y $15 \mathrm{dS} \mathrm{m}^{-1}$ que

${ }^{1}$ Cisneros-Saguilán, P., J. A. Saltijeral-Oaxaca y S. Vásquez-Agustín. 2003. La sustentabilidad de la ganadería bovina en la costa de Oaxaca. ICAPET. Oaxaca de Juárez, Oaxaca, México. 
corresponden a concentraciones teóricas de $0,1.28,2.56$, $3.84,6.40,7.68$ y $9.60 \mathrm{~g} \mathrm{~L}^{-1}$ de $\mathrm{NaCl}$, respectivamente. Cada tratamiento contó con seis repeticiones, dando un total de 42 unidades experimentales por cada cultivar, distribuidas en un diseño completamente al azar.

\section{Preparación de Soluciones}

Las soluciones se prepararon en laboratorio con base en la constante de proporcionalidad $\mathrm{mg}$ $\mathrm{L}^{-1}=640 \times \mathrm{CE}$ (Richards, 1985). Dónde: $\mathrm{mg} \mathrm{L}^{-1}=\mathrm{es}$ la concentración de las sales en solución; $\mathrm{CE}=$ es la conductividad eléctrica de la solución $\left(\mathrm{dS} \mathrm{m} \mathrm{m}^{-1}\right.$ a $\left.25^{\circ} \mathrm{C}\right)$. La CE y el pH se determinaron con un multiparámetro marca Hanna modelo HI 98129.

\section{Siembra}

En macetas con $2 \mathrm{~kg}$ de agrolita como sustrato, llevadas a capacidad de campo con un volumen de solución salina de $2.5 \mathrm{~L}$ por maceta, se sembraron 25 semillas por maceta para evaluar el porcentaje de emergencia de cada cultivar. En el caso del testigo se agregó solo agua destilada. Posteriormente, a los 15 días se realizó un aclareo para dejar tres plantas por maceta. Se regó con agua destilada cada tercer día, para reponer el agua consumida por evapotranspiración y mantener las unidades experimentales a peso constante. A la siembra y 14 días después, se aplicó al sustrato de las macetas el fungicida captan a dosis de $2 \mathrm{~g} \mathrm{~L}^{-1}$ para prevenir enfermedades. Para la nutrición de los pastos, a las macetas se les suministró un fertilizante universal soluble en agua (Miracle-Grow) con fórmula 15-30-15 a dosis de $3 \mathrm{~g} \mathrm{~L}^{-1}$. El experimento tuvo una duración de dos meses (del 5 de mayo al 5 de julio de 2018). Durante este periodo se realizó el conteo de plántulas emergidas cada tercer día en un lapso de 15 días, para tal efecto se consideró el coleóptilo (primera hoja visible arriba del sustrato). Cada semana se midió la longitud y diámetro del tallo principal y se contó el número de hijuelos; la longitud del tallo se midió desde la superficie del sustrato hasta el ápice del tallo con una regla graduada en centímetros. El diámetro del tallo principal se midió en su base con un vernier digital marca ${ }^{\circledR}$ Truper. El conteo de número de hijuelos por planta se hizo a partir de la tercera semana, cuando inició el macollamiento de los pastos. El número de hojas se contó a los 28, 42 y 60 días después de la emergencia (DDE). A los 60 DDE se cosecharon las plantas, se separaron y disectaron en raíz, tallo y hojas. La longitud de la raíz se midió de la base hasta el ápice radicular, con una regla graduada. Para la obtención del peso seco se secaron los órganos de la planta en estufa a $65^{\circ} \mathrm{C}$ durante 48 horas.

\section{Análisis Estadístico}

El análisis de varianza se realizó con el paquete estadístico Statistical Analysis System (SAS 9.0) y la comparación de medias mediante la prueba de Tukey con $\alpha=0.05$. Los datos se ajustaron a un modelo polinomial de predicción de la biomasa en función de la CE de las soluciones. Igualmente se utilizó la ecuación de Maas y Hoffman (1977) para la predicción del rendimiento biológico de ambos pastos en función de los niveles salinos experimentales, esta ecuación es: $\mathrm{Y}=100-\mathrm{b}$ (ECs-A), donde: $\mathrm{Y}=$ rendimiento; $\mathrm{A}=$ umbral salino, $\mathrm{b}=$ porcentaje de reducción de biomasa por unidad de aumento de salinidad, $\mathrm{CE}_{\mathrm{s}}=$ conductividad eléctrica de la solución salina.

\section{RESULTADOS Y DISCUSIÓN}

\section{Efecto del $\mathrm{NaCl} y$ sus Concentraciones en el Porcentaje de Emergencia de Pastos Cayman y Cobra}

A medida que aumentó el nivel de salinidad disminuyó el porcentaje de emergencia en ambos pastos (Cuadro 1). Esta reducción fue mayor en el pasto Cayman en comparación con el pasto Cobra, lo que es notorio a los 15 días después de la siembra (DDS) al incrementar el nivel salino de 0 a $15 \mathrm{dS} \mathrm{m}^{-1}$. La reducción del porcentaje de emergencia en Cobra fue de $14.7 \%$ mientras que en Cayman fue de $29.3 \%$. El análisis estadístico indica que en el pasto Cayman los dos últimos niveles de salinidad 12 y $15 \mathrm{dS} \mathrm{m}^{-1}$ produjeron un porcentaje de emergencia menor a $45 \%$, por lo que son estadísticamente diferentes a los otros niveles salinos $(P=0.05)$. En el caso del pasto Cobra la $\mathrm{CE}$ de $15 \mathrm{dS} \mathrm{m}^{-1}$ presentó una emergencia de $64.67 \%$ que es estadísticamente inferior al resto de los tratamientos $(P=0.05)$. La disminución de la emergencia de las plántulas en ambas especies se debe a que la sal $\mathrm{NaCl}$ causa en la semilla un estrés hídrico y osmótico provocado por una disminución del potencial hídrico del sustrato y la disminución de la disponibilidad de agua para la semilla, lo que dificulta su imbibición, por tanto, la semilla tiene que realizar 
Cuadro 1. Efecto de la sal $\mathrm{NaCl}$ y sus concentraciones sobre el porcentaje de emergencia de pastos Cayman y Cobra diversos días después de la siembra.

Table 1. Effect of $\mathrm{NaCl}$ salt and its concentrations on the percentage of emergence of Cayman and Cobra grasses several days after planting.

\begin{tabular}{|c|c|c|c|c|c|c|c|c|c|c|c|}
\hline \multirow{2}{*}{ Pasto } & \multirow{2}{*}{$\mathrm{CE}$} & \multirow{2}{*}{ Concentración } & \multirow{2}{*}{$\Psi \pi$} & \multirow{2}{*}{$\mathrm{pH}$} & \multicolumn{7}{|c|}{ Porcentaje de emergencia } \\
\hline & & & & & 3 & 5 & 7 & 9 & 11 & 13 & 151 \\
\hline \multirow{8}{*}{ Cayman B1* } & $\mathrm{dS} \mathrm{m} \mathrm{m}^{-1}$ & $\mathrm{~g} \mathrm{~L}^{-1}$ & $\mathrm{MPa}$ & \multicolumn{8}{|c|}{$\ldots \ldots-\ldots$ DDS $\ldots \ldots-\ldots$} \\
\hline & 0 & 0 & 0 & 7.01 & 5.33 & 46 & 65.33 & 70 & 70.67 & 71.33 & $71.33 \mathrm{a} /{ }^{2}$ \\
\hline & 2 & 1.28 & -0.07 & 6.53 & 2.67 & 38.67 & 60 & 68.67 & 69.33 & 69.33 & $70.00 \mathrm{a}$ \\
\hline & 4 & 2.56 & -0.14 & 6.37 & 3.33 & 38 & 53.33 & 58.67 & 60.67 & 64 & $64.67 \mathrm{a}$ \\
\hline & 6 & 3.84 & -0.21 & 6.35 & 0 & 34 & 56 & 62.67 & 63.33 & 62.67 & $64.00 \mathrm{a}$ \\
\hline & 10 & 6.4 & -0.36 & 6.39 & 0 & 25.33 & 45.33 & 57.33 & 58.67 & 61.33 & $60.67 \mathrm{ab}$ \\
\hline & 12 & 7.68 & -0.43 & 6.38 & 0 & 18 & 40.67 & 45.33 & 46 & 44.67 & $44.67 \mathrm{bc} *$ \\
\hline & 15 & 9.6 & -0.54 & 6.32 & 0 & 4 & 25.33 & 33.33 & 36 & 42 & $42 c^{*}$ \\
\hline & 0 & 0 & 0 & 7.01 & 22.67 & 61.33 & 74.67 & 76 & 77.33 & 79.33 & $79.33 \mathrm{a}$ \\
\hline & 2 & 1.28 & -0.07 & 6.53 & 26 & 66 & 74 & 75.33 & 75.33 & 75.33 & $76 a b$ \\
\hline & 4 & 2.56 & -0.14 & 6.37 & 11.33 & 48.67 & 70.67 & 74.67 & 76 & 75.33 & 76.67ab \\
\hline Cobra A & 6 & 3.84 & -0.21 & 6.35 & 19.33 & 59.33 & 75.33 & 79.33 & 79.33 & 78.67 & $78.67 \mathrm{ab}$ \\
\hline & 10 & 6.4 & -0.36 & 6.39 & 6 & 56.67 & 74 & 79.33 & 79.33 & 77.33 & 77.33ab \\
\hline & 12 & 7.68 & -0.43 & 6.38 & 12 & 42.67 & 57.33 & 61.33 & 64 & 66.67 & $66.0 \mathrm{ab}$ \\
\hline & 15 & 9.6 & -0.54 & 6.32 & 2.67 & 28.67 & 48.67 & 60.67 & 64 & 64.67 & $64.67 b^{*}$ \\
\hline
\end{tabular}

$\Psi \pi=$ potencial osmótico; DDS: días después de la siembra; ${ }^{1} \mathrm{~A}, \mathrm{~B}=$ prueba de medias entre pastos para la variable emergencia a los $15 \mathrm{DDS} ;{ }^{2} \mathrm{a}, \mathrm{b}, \mathrm{c}=$ prueba de medias entre niveles de CE para la variable emergencia a los 15 DDS. Medias con la misma letra en la misma columna no son estadísticamente diferentes, * significancia estadística (Tukey $\alpha=0.05$ ).

$\Psi \pi=$ osmotic potential; DDS: days after planting; ${ }^{1} \mathrm{~A}, \mathrm{~B}=$ test of means between pastures for the emergency variable at $15 \mathrm{DDS} ;{ }^{2} \mathrm{a}, \mathrm{b}, \mathrm{c}=$ test of means between $\mathrm{CE}$ levels for the emergency variable at 15 DDS. Means with the same letter in the same column are not statistically different, * statistical significance (Tukey $\alpha=0.05)$.

procesos de ajuste osmótico para superar el potencial del medio en el que está inmersa y así germinar; de lo contrario se deshidrata (Can-Chulim et al., 2014). Por otra parte, el exceso de iones de sodio y cloro tienden a causar daño en la membrana plasmática e intumescencia protoplásmica, que afectan la actividad enzimática y una producción inadecuada de energía por alteración en la respiración, así como la inhibición de la movilización de las reservas seminales hacia el eje embrionario, lo cual afecta el proceso germinativo y la emergencia (Laynez-Garzabal et al., 2007; Flowers et al., 2010). En el pasto Cayman se presentó un ligero retraso en la emergencia a CE de 6,10,12 y $15 \mathrm{dS} \mathrm{m}^{-1}$, ya que las semillas iniciaron emergencia 5 DDS a diferencia de los niveles menos salinos que indujeron la emergencia de plántulas 3 DDS. Esto se puede explicar en función del déficit hídrico y la toxicidad iónica que inhiben la síntesis de nitrato reductasa y las poliaminas que alteran los niveles de hormonas vegetales (mayor producción de ácido abscísico que de giberelinas), y deshidratan o desprenden el protoplasma de la pared celular, todo lo cual incrementa el periodo de quiescencia (Sánchez-Bernal y Ortega-Escobar, 2011). En este sentido González (2001) indica que el estrés hídrico y la acumulación de electrolitos en las células del embrión y luego en la plántula aminoran su crecimiento o incluso producen su muerte. En el experimento se pudo observar la muerte de plántulas en los dos pastos 11 DDS producido por los niveles salinos más altos. Los resultados son similares a los reportados por Bazzigalupi et al. (2008) en pasto agropiro alargado (Thinopyrum ponticum cv. Hulk), quienes determinaron 
que la germinación disminuyó en relación al testigo en 4,18 y $61 \%$ con incrementos de $\mathrm{NaCl}$ equivalentes a 6 , 12 y $18 \mathrm{dS} \mathrm{m}^{-1}$, respectivamente. Por su parte González et al. (2011) reportan que la germinación del pasto Banderita en $\mathrm{NaCl}$ fue seriamente afectado a partir de $12 \mathrm{dS} \mathrm{m}^{-1}$.

\section{Efecto de $\mathrm{NaCl} y$ sus Concentraciones en el Crecimiento de los Pastos Cayman y Cobra}

En general, en ambos pastos se presentó una reducción de la altura y diámetro del tallo, número de hijuelos, número de hojas y longitud de raíz conforme aumentó el nivel salino inducido por la sal $\mathrm{NaCl}$ (Cuadro 2).

En el pasto Cayman, la altura del tallo, el número de hijuelos y el número de hojas a $\mathrm{CE} \geq 10 \mathrm{dS} \mathrm{m}^{-1}$ presentaron reducciones significativas respecto al testigo $(P=0.05)$. Por su parte la raíz fue más sensible, las $C E \geq 6 \mathrm{dS} \mathrm{m}^{-1}$ la afectaron significativamente. En el caso del pasto Cobra, la altura, el número de hojas y la longitud de raíz presentaron decrecimiento significativo a $\mathrm{CE} \geq 12 \mathrm{dS} \mathrm{m}^{-1} \operatorname{con}(P=0.05)$. El número de hijuelos también fue afectado a CE de $15 \mathrm{dS} \mathrm{m}^{-1}$.

\section{Altura del Tallo de Plantas}

En pasto Cayman a $\mathrm{CE} \geq 10 \mathrm{dS} \mathrm{m}^{-1}$ y en pasto Cobra a $\mathrm{CE} \geq 12 \mathrm{dS} \mathrm{m}^{-1}$, la altura del tallo decreció $>$ $36 \%$ respecto al testigo $\left(\mathrm{CE}=0 \mathrm{dS} \mathrm{m}^{-1}\right)$. Esto dio como resultado alturas menores de $35 \mathrm{~cm}$ en ambos pastos. Tal disminución en altura a elevados niveles salinos se debe fundamentalmente al efecto de los factores osmótico, toxico-iónico y al desbalance nutricional, que inducen a nivel celular disminución de la producción de protoplasma, de división celular y de elongación que

Cuadro 2. Efecto de la sal $\mathrm{NaCl}$ y sus concentraciones en el crecimiento de los pastos Cayman y Cobra, 60 DDE.

Table 2. Effect of $\mathrm{NaCl}$ salt and its concentrations on the growth of Cayman and Cobra grasses, $60 \mathrm{DDE}$.

\begin{tabular}{|c|c|c|c|c|c|c|}
\hline Pasto & $\begin{array}{l}\text { Condutividad } \\
\text { eléctrica }\end{array}$ & Altura de tallo ${ }^{1}$ & Diámetro de tallo ${ }^{2}$ & $\begin{array}{l}\text { Número de } \\
\text { hijuelos }^{3}\end{array}$ & $\begin{array}{l}\text { Número de } \\
\text { hojas }^{4}\end{array}$ & Longitud de raíz ${ }^{5}$ \\
\hline \multirow{8}{*}{$\begin{array}{l}\text { Cayman } \\
\mathrm{A} 1, \mathrm{~A} 2, \mathrm{~A} 3, \mathrm{~A} 4, \mathrm{~B} 5 *\end{array}$} & $\mathrm{dS} \mathrm{m}^{-1}$ & $\mathrm{~cm}$ & $\mathrm{~mm}$ & & & $\mathrm{~cm}$ \\
\hline & 0 & $54.50 \mathrm{a} /{ }^{6}$ & $3.28 \mathrm{a} / 7$ & $13.17 \mathrm{a} /{ }^{8}$ & $43.67 \mathrm{a} /{ }^{9}$ & $39.75 \mathrm{a} /{ }^{10}$ \\
\hline & 2 & $53.67 \mathrm{a}$ & $3.17 \mathrm{a}$ & $11.33 \mathrm{a}$ & $39.17 \mathrm{a}$ & $29.50 \mathrm{ab}$ \\
\hline & 4 & $52.83 \mathrm{a}$ & $2.91 \mathrm{ab}$ & $11.83 \mathrm{a}$ & $42.33 \mathrm{a}$ & $33.83 \mathrm{ab}$ \\
\hline & 6 & $51.00 \mathrm{a}$ & $3.09 \mathrm{ab}$ & $8.50 \mathrm{ab}$ & $31.50 \mathrm{ab}$ & $25.67 b c^{*}$ \\
\hline & 10 & $35.17 b^{*}$ & $2.99 \mathrm{ab}$ & $5.67 b^{*}$ & $19.00 b^{*}$ & $22.17 b c^{*}$ \\
\hline & 12 & $26.17 b^{*}$ & $2.25 b^{*}$ & $3.50 b^{*}$ & $14.50 \mathrm{~b} *$ & $23.33 \mathrm{bc} *$ \\
\hline & 15 & $33.67 b^{*}$ & $2.93 \mathrm{ab}$ & $5 b^{*}$ & $16 b^{*}$ & $14.83 c^{*}$ \\
\hline \multirow{7}{*}{$\begin{array}{l}\text { Cobra } \\
\text { A1, A2, B3*, A4, A5 }\end{array}$} & 0 & $56.83 \mathrm{a}$ & $3.13 \mathrm{a}$ & $7.83 \mathrm{ab}$ & $34.00 \mathrm{a}$ & $39.33^{\mathrm{a}}$ \\
\hline & 2 & $50.25 \mathrm{ab}$ & $3.37 \mathrm{a}$ & $6.83 \mathrm{ab}$ & $31.33 \mathrm{ab}$ & $46.17^{\mathrm{a}}$ \\
\hline & 4 & $54.17 \mathrm{a}$ & $3.32 \mathrm{a}$ & $7.50 \mathrm{ab}$ & $30.83 \mathrm{ab}$ & $41.67 \mathrm{a}$ \\
\hline & 6 & $50.08 \mathrm{ab}$ & $3.54 \mathrm{a}$ & $8.33 \mathrm{a}$ & $31.83 \mathrm{ab}$ & $36.25 \mathrm{a}$ \\
\hline & 10 & $50 \mathrm{ab}$ & $3.18 \mathrm{a}$ & $7.17 \mathrm{ab}$ & $30 \mathrm{ab}$ & $37.75^{\mathrm{a}}$ \\
\hline & 12 & $33.83 \mathrm{bc}^{*}$ & $2.75 \mathrm{a}$ & $4.67 \mathrm{ab}$ & $15.83 \mathrm{bc} *$ & $17.77 b^{*}$ \\
\hline & 15 & $30.58 c^{*}$ & $2.79 \mathrm{a}$ & $3.50 b^{*}$ & $12.17 \mathrm{c}^{*}$ & $13.50 b^{*}$ \\
\hline
\end{tabular}

$\mathrm{A}, \mathrm{B}=$ prueba de medias entre pastos para las variables: ${ }^{1}$ altura de plantas, ${ }^{2}$ diámetro de tallo, ${ }^{3}$ número de hijuelos, ${ }^{4}$ número de hojas y ${ }^{5}$ longitud de raíz a 60 DDE. a, b, c $=$ prueba de medias entre niveles de CE para las variables: ${ }^{6}$ altura de plantas, ${ }^{7}$ diámetro de tallo, ${ }^{8}$ número de hijuelos, ${ }^{9}$ número de hojas y ${ }^{10}$ longitud de raíz a 60 DDE. Medias con la misma letra no son estadísticamente diferentes, * significancia estadística (Tukey $\alpha=0.05$ ).

$\mathrm{A}, \mathrm{B}=$ test of means between grasses for the variables: ${ }^{1}$ plant height, ${ }^{2}$ stem diameter, ${ }^{3}$ number of shoots, ${ }^{4}$ number of leaves and ${ }^{5}$ root length at 60 DDE. a, b, c $=$ test of means between CE levels for the variables: ${ }^{6}$ plant height, ${ }^{7}$ stem diameter, ${ }^{8}$ number of shoots, ${ }^{9}$ number of leaves and ${ }^{10}$ root length at 60 DDE. Means with the same letter are not statistically different, ${ }^{*}$ statistical significance (Tukey $\alpha=0.05$ ). 
limitan el crecimiento de los órganos vegetales (Lamz y González, 2013; Ramírez-Suárez y Hernández-Olivera, 2016). En condiciones de estrés las plantas disminuyen la concentración de fitohormonas tales como auxinas que actúan sobre la división celular y las giberelinas que actúan sobre la diferenciación, por lo que se atrofia el crecimiento de las plantas (Rodríguez-Pérez, 2006). En opinión de Yang et al. (1993), las auxinas favorecen el crecimiento del tallo, de tal forma que su inhibición llevará a una reducción de la expansión longitudinal de este órgano. Al respecto Laynez-Garzabal et al. (2008), reportan una disminución de la altura en gramíneas bajo condiciones salinas, por ejemplo, en plántulas de maíz alcanzó una altura de $26.75 \mathrm{~cm}$ a una $\mathrm{CE}$ de $0 \mathrm{dS} \mathrm{m} \mathrm{m}^{-1} \mathrm{y}$ de $8.03 \mathrm{~cm}$ a CE de $15 \mathrm{dS} \mathrm{m}^{-1}, 12 \mathrm{DDS}$. Por su parte Salomón y Samudio (2015) registran una disminución de altura de los pastos Panicum maximum Jacq. variedades Tanzania y Mombasa a medida que aumenta la CE de 0 a $22 \mathrm{dS} \mathrm{m}^{-1}$. De la misma forma Argentel et al. (2006) observaron en Triticum aestivum L. variedad Cuba-c-204 una disminución de la altura de 33 y $35 \%$ al incrementar la CE a niveles de 25 y $28 \mathrm{dS} \mathrm{m}^{-1}$ respecto al testigo. Los resultados de la presente investigación confirman que la altura de tallo de los pastos Cayman y Cobra, fue afectada de manera diferencial por los máximos niveles salinos.

\section{Diámetro del Tallo Principal}

Los resultados indican que en pasto Cayman la $\mathrm{CE}$ de $12 \mathrm{dS} \mathrm{m}^{-1}$ produjo en las plantas la máxima disminución en diámetro del tallo comparado con el efecto producido en los otros niveles salinos.

Esto se puede explicar en función de un crecimiento rápido poco sostenido que efectúan las plantas de pasto a $\mathrm{CE}>12 \mathrm{dS} \mathrm{m}^{-1}$. En el caso del pasto Cobra no se presentaron diferencias estadísticas, aunque es posible notar en los dos pastos que a CE de $\left(0\right.$ a $\left.10 \mathrm{dS} \mathrm{m}^{-1}\right)$ se registraron valores $>3 \mathrm{~mm}$ de diámetro del tallo $\mathrm{y}$ a una $\mathrm{CE} \geq 12 \mathrm{dS} \mathrm{m}^{-1}$ los diámetros fueron de menor magnitud. Los bajos valores obtenidos en niveles más salinos, se explican por la pérdida de turgencia que provocó la salinidad por efecto osmótico en las células del tejido vascular. Un estudio realizado por Luna-Flores et al. (2012) indican una reducción de contenido de agua del tallo de 28, 59 y 60\% en Cordia dodecandra A. DC., Piscidia piscipula (L.) Sarg. y
Leucaena leucocephala (Lam.), respectivamente, sometidas a estrés hídrico, en relación a las que no fueron sometidas a dicho estrés. El contenido de agua es esencial para la expansión y elongación celular ya que cuando las células pierden turgencia, no llevan a cabo este proceso (Rodríguez-Pérez, 2006; Moreno, 2009), lo que afecta directamente al diámetro del tallo. Es importante señalar que investigaciones de Strogonov (1964) indican que diversas gramíneas sometidas a $\mathrm{CE}>6 \mathrm{dS} \mathrm{m}^{-1}$, produjeron una disminución del grosor de la pared celular y del tamaño de células meristemáticas del cambium del tejido vascular.

\section{Número de Macollos}

El número de macollos disminuyó conforme aumenta la CE. El pasto Cayman presentó mayor número de macollos que el pasto Cobra a $\mathrm{CE}$ de 0 a $4 \mathrm{dS} \mathrm{m} ~^{-1}$. Sin embargo, éste último tiene mayor tolerancia a condiciones con mayor concentración de sal. El número de macollos está determinado por la tasa de formación de hojas, ya que cada hoja presenta una yema axilar que puede dar origen a un nuevo macollo (Borrajo y Alonso, 2015). En condiciones salinas se inhibe el crecimiento de yemas axilares del tallo de pastos que dan origen a los macollos (Munns y Tester, 2008), esto disminuye su número por planta, lo que se puede observar en los resultados obtenidos. En efecto, ambos pastos a concentraciones de sal $>7.68 \mathrm{~g} \mathrm{~L}^{-1}$, registraron menos de cinco macollos 60 DDE. La salinidad causa numerosos problemas para el crecimiento de las plantas. Ante al estrés, las plantas responden con una variedad de mecanismos para sobrevivir; en caso de una defoliación extrema las gramíneas envían los fotoasimilados producidos en las hojas remanentes y cesa la exportación de fotoasimilados a yemas axilares para formación de macollos (Saroff et al., 2003). Esta situación podría darse en los pastos sometidas a las condiciones salinas experimentales. Investigaciones realizadas por Worku et al. (2019) en pasto Rhodes (Chloris gayana Kunth), indican reducción de numero de macollos por planta de 7.2, 7.1, 6.6, 5.9 y 4.8 a CE de $0,5,10,15$ y $20 \mathrm{dS} \mathrm{m}^{-1}$ respectivamente. Igualmente, Yapu y Yapu (2014), registraron en cebada forrajera 739 tallos $\mathrm{m}^{-1}$ a CE de $0.94 \mathrm{dS} \mathrm{m}^{-1}$ y disminuyó a 340 tallos $\mathrm{m}^{-1}$ a CE de $8.76 \mathrm{dS} \mathrm{m}^{-1}, 110 \mathrm{DDS}$. 


\section{Número de Hojas}

El pasto Cayman a $\mathrm{CE} \geq 10 \mathrm{dS} \mathrm{m}^{-1}$ registró una reducción del número de hojas mayor del $50 \%$ y en pasto Cobra esta reducción se presentó a $\mathrm{CE} \geq 12 \mathrm{dS} \mathrm{m}^{-1}$, lo que denota diferencia de tolerancia a la sal $\mathrm{NaCl}$. La reducción del número de hojas en las plantas sometidas a estrés salino se debe a la excesiva absorción del ion $\mathrm{Na}^{+}$al interior de las células meristemáticas, esto eleva su concentración dentro del protoplasma que provoca inhibición del crecimiento celular por plasmólisis, un efecto que puede ser reversible o permanente lo que depende de la periodicidad del abastecimiento de agua mediante riego, y que a su vez influye en la intensidad y la duración del estrés, que en casos extremos puede causar necrosis en los tejidos vegetales (Strogonov, 1964; Sánchez-Bernal et al., 2013). La causa de la necrosis es probablemente por la concentración excesiva de sales que excede la habilidad de la célula de compartimentar sales en la vacuola (Lamz y Cepero, 2013). Otros efectos causados por la sal en las hojas son el cierre de estomas, la reducción del número de estomas y el aumento en el espesor del tejido foliar para evitar la transpiración (Parés et al., 2008). En este sentido, Martínez et al. (2011) sugiere que el estrés hídrico causado por la salinidad se manifiesta en la reducción de expansión foliar y pérdida de turgencia. En pasto gigante (Miscanthus $x$ giganteus J. M. Greef \& Deuter ex Hodk. \& Renvoize) a CE $>2.86 \mathrm{dS} \mathrm{m}^{-1} \mathrm{se}$ registró una disminución del número de hojas respecto al testigo y a CE de $10.65 \mathrm{dS} \mathrm{m}^{-1}$ la reducción fue de $50 \%$ en número de hojas (Stavridou et al., 2017), datos similares a los obtenidos en este estudio. Por su parte Aiazzi et al. (2005) reportan disminución del número de hojas y área foliar en uno de los genotipos de Atriplex cordobensis Gandoger et Stuckert (Chenopodiaceae) en condiciones salinas a $\mathrm{CE}$ de $25 \mathrm{dS} \mathrm{m}^{-1}$.

\section{Longitud de Raíz}

Niveles salinos $>6 \mathrm{dS} \mathrm{m}^{-1}$, produjeron una reducción de la longitud de la raíz de pasto Cayman, mientras que la raíz de pasto Cobra redujo su longitud a $\mathrm{CE}>12 \mathrm{dS} \mathrm{m}^{-1}$. Esta respuesta diferencial de la longitud radicular se debe a la diferente tolerancia a salinidad de cada especie, como lo señalan (Mass y Hoffman, 1977). Asociado a lo anterior, están las perturbaciones fisiológicas que sufre la raíz en condiciones salinas como son la disminución de la producción de protoplasma, de división celular de las células meristemáticas y de elongación de las células de la zona de alargamiento arriba de la cofia que se atribuye al intenso efecto osmótico de esta sal (Ramos et al., 2004). Es importante analizar el efecto de la sal en la raíz, ya que se ha comprobado que en condiciones de estrés salino la raíz se convierte en un órgano de almacenamiento de sustancias de reserva que favorece el crecimiento de la parte aérea, de tal forma que en la medida que la raíz tolere más la sal, tendrá la capacidad de explorar más volumen de suelo para abastecerse de agua y nutrientes y mantener vivo al organismo vegetal (Strogonov, 1964). Esto tiene implicaciones importantes para que el sistema foliar continúe la fotosíntesis para la formación de fotoasimilados (Sánchez-Bernal y Ortega-Escobar, 2011; Guevara y Guenni, 2013). Así mismo la sal NaCl produce un deterioro en las propiedades de la pared celular por el desplazamiento del $\mathrm{Ca}^{2+}$ por el ion $\mathrm{Na}^{+}$, lo que disminuye la permeabilidad al agua y la síntesis de la pared celular, lo cual limita el crecimiento de la planta (Pritchard, 1994). Por su parte Sam (2007) reporta cambios de la forma y organización de los tejidos de la raíz en condiciones de salinidad, que impiden un óptimo desarrollo. Investigadores como Jauregui et al. (2017) reportaron en pasto Agropiro criollo (Elymus scabrifolius (Döll) J. H. Hunz.) una reducción en la longitud de raíz $>50 \%$ respecto al testigo cuando se les sometió a una $\mathrm{CE}$ de $27 \mathrm{dS} \mathrm{m} \mathrm{m}^{-1}$. Por su parte en pasto Banderita (Bouteluoa curtipendula (Michx.) Torr.), se determinó que a $\mathrm{CE}$ de $0 \mathrm{dS} \mathrm{m}^{-1}$ la longitud radicular alcanzó en promedio $10 \mathrm{~mm}$ y a CE de $15 \mathrm{dS} \mathrm{m}^{-1}$ disminuyó a menos de $2 \mathrm{~mm}$ e incluso a $\mathrm{CE}$ de $28 \mathrm{dS} \mathrm{m}^{-1}$ no se promovió crecimiento de este órgano 15 DDS (González et al., 2011). Estudios realizados por Taleisnik et al. (1997), señalan la disminución de la longitud de raíz conforme aumentó la concentración de la sal $\mathrm{NaCl}$ en pasto Rhodes cv. Boma, a partir de $10 \mathrm{dS} \mathrm{m}^{-1}$ que redujo el rendimiento $50 \%$ respecto al testigo. Estos resultados son congruentes con los obtenidos en la presente investigación.

\section{Efecto de la Sal $\mathrm{NaCl}$ y sus Concentraciones en la Biomasa Seca de los Pastos Cayman y Cobra 60 DDE}

De acuerdo a los resultados obtenidos, el peso seco de la parte aérea (tallo y hojas) y de la parte radicular disminuyó conforme aumentó la CE en el sustrato para 

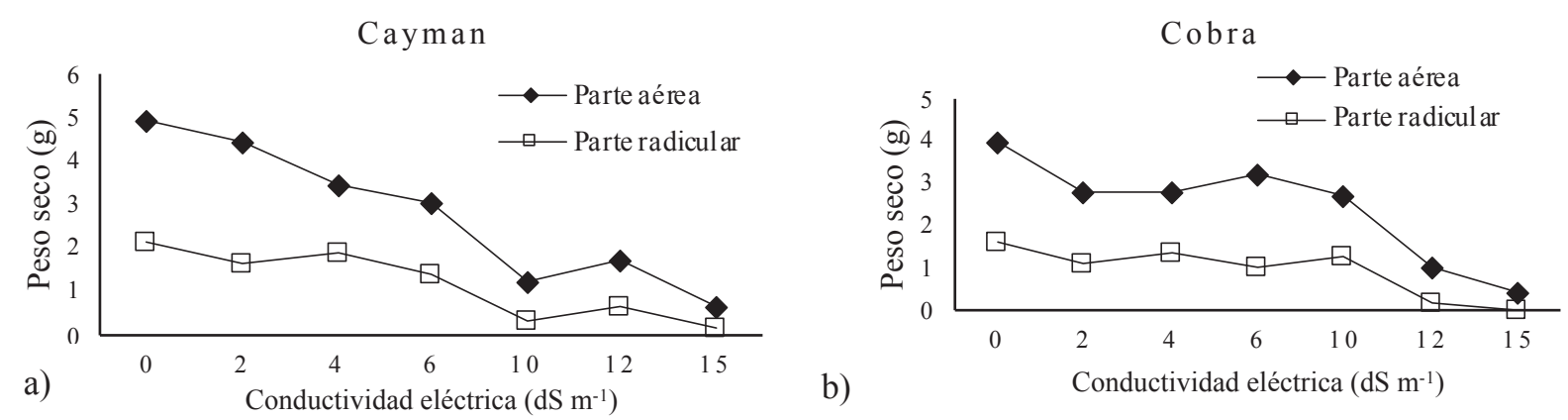

Figura 1. Efecto de la salinidad por $\mathrm{NaCl}$ en la acumulación de biomasa de la parte aérea y raíz del pasto a los $60 \mathrm{DDE}$. a) pasto Cayman; b) pasto Cobra.

Figure 1. Effect of $\mathrm{NaCl}$ salinity on the biomass accumulation of the aerial part and root of the grass at 60 DDE.

a) Cayman grass; b) Cobra grass.

ambos pastos (Figura 1). En niveles bajos $\left(<4 \mathrm{dS} \mathrm{m}^{-1}\right)$, el pasto Cayman mostró mayor acumulación de biomasa. En opinión de Strogonov (1964) esto se debe a que algunas especies vegetales, ante el estrés salino realizan un crecimiento rápido poco sostenido que les permite tolerar ese umbral salino, después de lo cual son vulnerables a niveles mayores de salinidad. El pasto Cobra presentó mayor tolerancia a niveles altos de salinidad $\left(>6 \mathrm{dS} \mathrm{m}^{-1}\right)$, ya que presentó menor reducción de biomasa en la parte aérea y de la raíz, la cual fue significativa $(P=0.05)$ respecto al testigo a $\mathrm{CE} \geq 12 \mathrm{dS} \mathrm{m}^{-1}$ en comparación con el pasto Cayman que redujo significativamente la producción de biomasa de la parte aérea y la raíz a $\mathrm{CE} \geq 10 \mathrm{dS} \mathrm{m}^{-1}$ esto indica que el pasto Cobra fue más tolerante a niveles salinos extremos.

El pasto Cayman a $\mathrm{CE} \geq 10 \mathrm{dS} \mathrm{m}^{-1}$, presentó en la parte aérea, una reducción del peso seco mayor al $40 \%$ y en raíz una reducción mayor al $33 \%$. El pasto Cobra a $\mathrm{CE} \geq 12 \mathrm{dS} \mathrm{m}^{-1}$, presentó en la parte aérea una reducción del peso seco mayor del $30 \%$ y en raíz se registró una reducción del peso seco mayor del $40 \%$. La disminución de la biomasa en plantas sometidas a estrés salino, es consecuencia de la reducción de la expansión foliar, altura y grosor de tallo y longitud de raíz, que afectó en forma adversa la biomasa acumulada en la parte aérea, así como en la raíz. Estudios realizados en condiciones de estrés salino a CE de la solución de suelo de $9.6 \mathrm{dS} \mathrm{m}^{-1}$, durante 21 días, en diversas especies forrajeras como pasto Estrella (Cynodon nlemfluencis Vanderyst), registraron una reducción de $69.33 \%$ en el vástago y $62.44 \%$ en la raíz; en cebada forrajera cv Esmeralda (Hordeum vulgare L) de
$27.29 \%$ en el vástago y $18.81 \%$ en la raíz y en pasto Raygras (Lolium perenne L.) de 31.04\% en el vástago y de $37.18 \%$, en la raíz, respectivamente (Lastiri et al., 2017). La reducción de biomasa varía entre especies e incluso entre cultivares de la misma especie lo que se pudo constatar en esta investigación. De la misma forma Dai et al. (2009), reportan disminución de biomasa con el aumento de salinidad en pasto Azul (Poa annua L.) a CE de $1.2 \mathrm{dS} \mathrm{m}^{-1}$ ya que registraron 8.7 gramos por planta $\left(\mathrm{g} \mathrm{pl}^{-1}\right)$ que disminuyó a $3.9 \mathrm{~g} \mathrm{pl}^{-1}$ a $15 \mathrm{dS} \mathrm{m}^{-1}$.

En condiciones salinas de $\mathrm{NaCl}$, el comportamiento del peso seco total de las plantas de pastos Cayman y Cobra se ajusta a los modelos de la ecuación cuadrática señalados en la Figura 2.

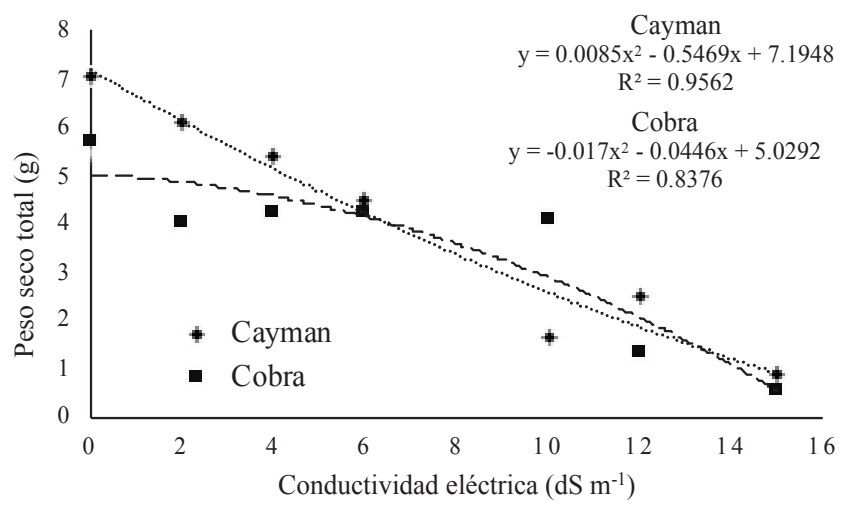

Figura 2. Peso seco total de los pastos Cayman y Cobra en función de la conductividad eléctrica.

Figure 2. Total dry weight of Cayman and Cobra grasses depending on electrical conductivity. 


\section{Predicción del Rendimiento Biológico de los Pastos Cayman y Cobra en Condiciones Salinas Inducidas por $\mathrm{NaCl}$}

La raíz, el tallo y las hojas, son órganos de interés para la producción de forraje de los pastos Cayman y Cobra, ya que su crecimiento adecuado está íntimamente asociado a la producción de biomasa para alimentación animal. En lo fundamental ambos pastos presentaron una tendencia a disminuir el peso seco total a medida que incrementó la salinidad (Cuadro 3 ). La reducción de biomasa fue más pronunciada en pasto Cayman que en pasto Cobra, ya que a $\mathrm{CE}>2 \mathrm{dS} \mathrm{m}^{-1}$, el pasto Cayman disminuyó $0.4 \mathrm{~g} \mathrm{pl}^{-1}$, en tanto que el pasto Cobra disminuyó $0.3 \mathrm{~g} \mathrm{pl}^{-1}$ por cada unidad que aumentó la CE. Otras investigaciones realizadas registran una disminución de biomasa en condiciones de estrés salino. En pasto gigante (Miscanthus $x$ giganteus) se presentó una reducción de $56 \%$ en un intervalo del 5 a $15 \mathrm{dS} \mathrm{m}^{-1}$ (Stavridou et al., 2017) y en festuca (Festuca arundinacea Schreb) una reducción de $30 \%$ a $8 \mathrm{dS} \mathrm{m}^{-1}$ (Kaplan et al., 2017). De acuerdo con la clasificación de Maas y Hoffman (1977), al considerar el porcentaje de reducción de biomasa en función de los niveles salinos, el pasto Cayman puede clasificarse como moderadamente sensible a la sal $\mathrm{NaCl}$ y el pasto Cobra como moderadamente tolerante.

\section{CONCLUSIONES}

- Los pastos Cayman y Cobra son afectados por la sal $\mathrm{NaCl}$ en su etapa de emergencia y etapas subsecuentes del desarrollo. A medida que aumenta la CE de las soluciones salinas, ambos pastos, disminuyeron de manera diferencial el porcentaje de emergencia, la altura y diámetro del tallo, el número de hijuelos, el número de hojas y la biomasa.

- De acuerdo a la clasificación de Maas y Hoffman (1977) el pasto Cayman se considera moderadamente sensible y el pasto Cobra moderadamente tolerante a la sal $\mathrm{NaCl}$.

\section{AGRADECIMIENTOS}

Se agradece a la empresa Semillas Papalotla S. A de C.V por el apoyo recibido para la realización de esta investigación.

\section{LITERATURA CITADA}

Aiazzi, M. T., P. Carpane y C. Deza. 2005. Efecto de la salinidad, sobre el crecimiento de plantas de Atriplex cordobensis Gandoger et Stuckert originadas de semillas de distintas procedencias. Multequina 14: 39-46.

Argentel, L., M. González y R. Plana. 2006. Efecto de altas concentraciones salinas sobre la germinación y el crecimiento del trigo (Triticum aestivum) variedad Cuba c-204. Cult. Trop. 27: $45-48$.

Bazzigalupi, O., S. M. Pistorale y A. N. Andrés. 2008. Tolerancia a la salinidad durante la germinación de semillas provenientes de poblaciones naturalizadas de agropiro alargado (Thinopyrum ponticum). Cienc. Inv. Agr. 35: 277-285. http://dx.doi. org/10.4067/S0718-16202008000300005.

Bizhani, S. and H. Salehi. 2014. Physio-morphological and structural changes in common bermudagrass and Kentucky bluegrass during salt stress. Acta Physiol. Plant. 36: 777-786. doi: https://doi.org/10.1007/s11738-013-1455-y.

Borrajo, C. I. y S. I. Alonso. 2015. Tasa de aparición de hojas y macollos en Thinopyrum ponticum, efecto del ambiente y el material genético. $38^{\circ}$ Congreso de la Asociación Argentina de Producción Animal. La Pampa, Argentina.

Cuadro 3. Reducción de biomasa de los pastos Cayman y Cobra en función de los niveles de CE.

Table 3. Reduction of biomass of Cayman and Cobra grasses according to EC levels.

\begin{tabular}{|c|c|c|c|c|c|c|c|c|c|}
\hline \multirow{2}{*}{ Pastos } & \multicolumn{3}{|c|}{ Intervalos de CE } & \multicolumn{3}{|c|}{ Reducción de biomasa } & \multicolumn{3}{|c|}{ Nivel de tolerancia } \\
\hline & Nivel 1 & Nivel 2 & Nivel 3 & Nivel 1 & Nivel 2 & Nivel 3 & 1 & 2 & 3 \\
\hline & $-\cdots$ & $\mathrm{dS} \mathrm{m}^{-1}$ & $-\ldots$ & $-\cdots$ & $-\%-$ & $-\ldots$ & & & \\
\hline Cayman & $(2,4]$ & $(4,10]$ & $(10,15]$ & 17.82 & 47.52 & 80.19 & $\mathrm{~T}$ & MS & MS \\
\hline Cobra & $(2,4]$ & $(4,10]$ & $(10,15]$ & 15.63 & 41.68 & 70.34 & $\mathrm{~T}$ & MS & MT \\
\hline
\end{tabular}

$\mathrm{T}=$ tolerante $; \mathrm{MS}=$ moderadamente sensible $; \mathrm{MT}=$ moderadamente tolerante.

$\mathrm{T}=$ tolerant $; \mathrm{MS}=$ moderately sensitive $; \mathrm{MT}=$ moderately tolerant. 
Can-Chulim, A., L. G. Ramírez-Guerrero, H. M. Ortega-Escobar, E. Cruz-Crespo, D. Flores-Román, E. I. Sánchez-Bernal y A. Madueño-Molina. 2014. Germinación y crecimiento de plántulas de Phaseolus vulgaris L. en condiciones de salinidad. Rev. Mex. Cienc. Agríc. 5: 753-763.

Cruz-Tejeda, J. M., J. V. Ray-Ramírez, J. L. Ledea-Rodríguez y R. C. Arias-Pérez. 2017. Establecimiento de nuevas variedades de Cenchrus purpureus en un ecosistema frágil del Valle del Cauto, Granma. Rev. Prod. Anim. 29: 29-35.

Dai, J., D. R. Huf, and M. J. Schlossberg. 2009. Salinity effects on seed germination and vegetative growth of greens-type Poa annua relative to other cool-season turfgrass species. Crop. Sci. 49: 696-703. doi: https://doi.org/10.2135/ cropsci2008.04.0221.

Flowers, T. J., P. M. Gaur, C. L. Gowda, L. Krishnamurthy, S. Samineni, K. H. Siddique, N. C. Turner, V. Vadez, R. K. Varshney, and T. D. Colmer. 2010. Salt sensitivity in chickpea. Plant Cell Environ. 33: 490-509. doi: https://doi.org/10.1111/ j.1365-3040.2009.02051.x.

González, L. M. 2001. Apuntes sobre la fisiología de las plantas cultivadas bajo estrés de salinidad. Cult. Trop. 23: 47-57.

González-Romero, S. L., A. R. Quero-Carrillo, O. Franco-Mora, C. Ramirez-Ayala, H. M. Ortega-Escobar y C. Trejo-López. 2011. Tolerancia a la salinidad del pasto Banderita (Bouteluoa curtipendula (Michx.) Torr.) en la etapa de germinación en dos regímenes de temperaturas. Cienc. Ergo Sum. 17: 277-285.

Guevara, E. y O. Guenni. 2013. Densidad y longitud de raíces en plantas de Leucaena leucocephala (Lam) De Wit. Multiciencias 13: 372-380.

Jauregui, C. G., M. A. Ruiz y R. D. Ernst. 2017. Tolerancia a la salinidad en plántulas de agropiro criollo (Elymus scabrifolius) y agropiro alargado (Thinopyron ponticum). Pastos y Forrajes 40: 29-36.

Kaplan, M., M. Baser, H. Kale, H. A. Irik, I. Ulger, and A. Unlukara. 2017. Change in yield and chemical composition of tall fescue (festuca arundinacea schreb.) plants under salt stress. Turkish J. Field Crops. 22: 204-210. doi: https://doi.org/10.17557/ tjfc. 356220 .

Lamz-Piedra, A. y M. C. González-Cepero. 2013. La salinidad como problema en la agricultura: la mejora vegetal una solución inmediata. Cult. Tropic. 34: 31-42.

Lastiri-Hernández, M. A., D. Álvarez-Bernal, L. H. Soria-Martínez, S. Ochoa-Estrada y G. Cruz-Cárdenas. 2017. Efecto de la salinidad en la germinación y emergencia de siete especies forrajeras. Rev. Mex. Cienc. Agríc. 8: 1245-1257.

Laynez-Garsaball, J. A., J. R. Méndez-Natera y J. Mayz-Figueroa. 2007. Efecto de la salinidad del suelo sobre la germinación de semillas de maíz de diferentes pesos en el oriente venezolano. Temas Agrarios 12: 62-73. doi: https://doi.org/10.21897/rta. v12i2.658.

Laynez-Garsaball, J. A., J. R. Méndez-Natera y J. Mayz-Figueroa. 2008. Efecto de la salinidad y del tamaño de la semilla sobre la germinación y crecimiento de plántulas de maíz (Zea mays L.) bajo condiciones de laboratorio. Rev. Esp. Cienc. Quím. Biol. 11: 17-25. doi: https://doi.org/10.22201/ fesz.23958723e.2008.1.7.

Luna-Flores, W., H. Estrada-Medina, J. J Jiménez-Osornio y L. L. Pinzón-López. 2012. Efecto del estrés hídrico sobre el crecimiento y eficiencia del uso del agua en plántulas de tres especies arbóreas caducifolias. Terra Latinoamericana 30: 343-353.
Maas, E. V. and G. J. Hoffman. 1977. Crop salt tolerance current assessment. J. Irrig. 103: 115-134.

Martínez-Villavicencio, N., C. V. López-Alonzo, M. Basurto-Sotelo y R. Pérez-Leal. 2011. Efectos por salinidad en el desarrollo vegetativo. Tecnociencia 5: 156-161.

Masters, D. G., S. E. Benes, and H. C. Norman. 2007. Biosaline agriculture for forage and livestock production. Agric. Ecosyst. Environ. 119: 234-248. doi: https://doi.org/10.1016/j. agee.2006.08.003.

Mesa, D. 2003. Obtención de plantas resistentes a la salinidad para los suelos salinos cubanos. Rev. Cubana Cienc. Agríc. 37: $217-$ 226.

Moreno F., L. P. 2009. Respuesta de las plantas al estrés por déficit hídrico. Agron. Colomb. 27: 179-191.

Munns, R. and M. Tester. 2008. Mechanisms of salinity tolerance. Annu. Rev. Plant Biol. 59: 651-681. doi: https://doi. org/10.1146/annurev.arplant.59.032607.092911.

Nizam, I. 2011. Effect of salinity stress on water uptake, germination and early seedling growth of perennial ryegrass. Afr. J. Biotechnol. 10: 10418-10424. doi: https://doi.org/10.5897/ AJB11.1243.

Parés, J., M. Arizaleta, M. E. Sanabria y G. García. 2008. Efecto de los niveles de salinidad sobre la densidad estomática, índice estomático y el grosor foliar en plantas de Carica papaya L. Acta Bot. Venez. 31: 27-34.

Pritchard, J. 1994. The control of cell expansion in roots. New Phytol. 68: 3-26. doi: https://doi.org/10.1111/j.1469-8137.1994. tb04255.x.

Ramírez-Suárez, W. y L. A. Hernández-Olivera. 2016. Tolerancia a la salinidad en especies cespitosas. Pastos y Forrajes. 39: 235245.

Ramos, J. C., M. G. Perreta, J. C. Tivano y A. C. Vegetti. 2004. Variaciones anatómicas en la raíz de Pappophorum philippianum inducidas por salinidad. Rev. Int. Bot. Exp. 73: 103-109.

Richards, L. A. 1985. Diagnóstico y rehabilitación de suelos salinos y sódicos. Laboratorio de Salinidad del Departamento de Agricultura de los E. U. A. Manual 60. Limusa. México, D. F.

Rodríguez-Pérez, L. 2006. Implicaciones fisiológicas de la osmorregulación en plantas. Agron. Colomb. 24: 28-37.

Ruiz-Cerda, E., R. A. Aldaco-Nuncio, J. A. Montemayor-Trejo, M. Fortis-Hernández, J. Olague-Ramírez y J. C. VillagomezGamboa. 2007. Aprovechamiento y mejoramiento de un suelo salino mediante el cultivo de pastos forrajeros. Téc. Pec. Méx. 45: 19-24.

Ruiz-Ramírez, S., A. Valdés-Oyervides, F. Facio-Parra y L. ArceGonzález. 2012. Efecto de diferentes niveles de salinidad en la germinación y vigor de semillas de cinco gramíneas forrajeras. Agraria 9: 7-13.

Salomón, J. y A. Samudio. 2015. Efecto del estrés salino en la germinación y vigor de semillas de Panicum maximum Jacq. variedades Tanzania y Mombasa. Compend. Cienc. Vet. 5: 23-31. doi: http://dx.doi.org/10.18004/compend.cienc. vet.2015.05.02.23-31.

Sam, O. 2007. Influencia de la salinidad en las radículas de plántulas de arroz (Oryza sativa). Cult. Tropic. 28: 25-28.

Sánchez-Bernal, E. I. y H. M. Ortega-Escobar. 2011. Emergencia de brotes de tubérculos de papa en condiciones salinas. Terra Latinoamericna 29: 153-160. 
Sánchez-Bernal, E. I., H. M. Ortega-Escobar, G. T. SandovalOrozco, R. A. Hernández-Viruel y C. Estrada-Vázquez. 2012a. Lavado de sales en suelo aluviales costeros de Oaxaca, México, con aguas residuales municipales tratadas. Rev. Int. Contam. Ambient. 28: 343-360.

Sánchez-Bernal, E. I., M. Camacho-Escobar, A. RodríguezLeón, and H. M. Ortega-Escobar. 2012b. Characterization of soils affected by salts in irrigation district 110 "Rio Verde", Oaxaca, Mexico. Afr. J. Agric. Res. 7: 943-957. doi: https:// doi.org/10.5897/AJAR11.1479.

Sánchez-Bernal, E. I., M. A. Camacho-Escobar, A. RodríguezLeón, and H. M. Ortega-Escobar. 2013. Physiological behavior of potato cv. Tollocan at diverse types of salinity. J. Plant Stud. 2: 120-134. doi: https://doi.org/10.5539/jps.v2n1p120.

Sánchez-Bernal, E. I., G. Sandoval-Orozco, M. A. CamachoEscobar, F. Valdez-Martínez, A. Rodríguez-León y H. M. Ortega-Escobar. 2014. Calidad hidrogeoquímica de las aguas del río Copalita, Oaxaca, México. Rev. Int. Cienc. Soc. 1: $27-41$.

Sánchez-Bernal, E. I., H. M. Ortega-Escobar, E. S. Leal-Pérez, A. Can-Chulim, V. Ortega-Baranda, M. A. Camacho-Escobar, and O. Mancilla-Villa. 2019. Interannual salinity in a coastal lagoon of Oaxaca, Mexico: Effects on growth of black mangrove. Transylvanian Rev. 27: 9240-9256.

Saroff, C., H. Pagliaricci y V. Ferreira. 2003. Efecto de la defoliación sobre la dinámica del crecimiento de triticale. Agric. Téc. 63: 266-276. doi: http://dx.doi.org/10.4067/S036528072003000300006.
Serrano-Altamirano, V., M. M. Silva-Serna, M. A. Cano-García, G. Medina-García y A. Ruiz-Coral. 2005. Estadísticas climatológicas básicas del Estado de Oaxaca (Periodo 19612003). INIFAP, SAGARPA. Libro técnico $N^{\circ}$ 4. ISBN: 97043-0007-7.

Stavridou, E., A. Hastings, R. J. Webster, and P. H. Robson. 2017. The impact of soil salinity on the yield, composition and physiology of the bioenergy grass Miscanthus $x$ giganteus. Global Change Biol. Bioener. 9: 92-104. doi: https://doi. org/10.1111/gcbb.12351.

Strogonov, B. P. 1964. Physiological basis of salt tolerance of plants. (As affected by various types of salinity). Jerusalem, Israel Program for Scientific Translations. Jerusalem, Israel.

Taleisnik, E., G. Peyrano, and C. Arias. 1997. Response of Chloris gayana cultivars to salinity. 1 . Germination and early vegetative growth. Trop. Grass. 31: 232-240.

Worku-Daba, A., A. Sarwar-Qureshi, and B. Nekir-Nisaren. 2019. Evaluation of some Rhodes grass (Chloris gayana) genotypes for their salt tolerance, biomass yield and nutrient composition. Appl. Sci. 9: 1-12. doi: https://doi.org/10.3390/app9010143.

Yang, T., D. M. Law, and P. J. Davies. 1993. Magnitude and kinetics of stem elongation induced by exogenous Indole-3-acetic acid in intact light rown Pea seedlings. Plant Physiol. 102: 707-724. doi: https://doi.org/10.1104/pp.102.3.717.

Yapu, G. y W. Yapu. 2014. Efecto de la salinidad sobre el rendimiento de tres variedades de cebada forrajera. IINEA Mollesnejta 1: 1-8. 